\title{
A comparative study of CBP, CRP, Micro ESR and blood culture in the diagnosis of neonatal septicemia
}

\author{
Mir Sumsam Ali Khurram ${ }^{1}$, Naseem A $A^{2}$, Prasad C.N. D \\ ${ }^{1}$ Dr. Mir Sumsam Ali Khurram, Associate Professor, Department of Pediatrics, Deccan College of Medical Sciences, \\ Hyderabad, Telangana State, India, ${ }^{2}$ Dr. Altaf Naseem, Professor Department of Pediatric, Deccan College of Medical \\ Sciences, Hyderabad, Telangana State, India, ${ }^{3}$ Dr. C. N. Prasad, Professor \& Head of the Department of Pediatric \\ Surgery, Deccan College of Medical Sciences, Hyderabad, Telangana State, India.
}

Address for Corresponence: Dr. Mir Sumsam Ali Khurram, Associate Professor, Department of Pediatrics, Deccan College of Medical Sciences, Hyderabad, Telangana State, India. E-mail: dr.sakhurram@gmail.com

\begin{abstract}
Background: Neonatal sepsis is one of the most important causes of morbidity and mortality in children. The incidence was 3\% in intramural births, with an overallmortality rate of variation of 18.6\%. Aims and Objectives: To evaluate the role of blood culture, micro ESR, CBP, CRP in the diagnosis of sepsis in children. To study sensitivity, specificity, positive predictive values and negative predictive values of the above hematological parameters, either individually or in combination with diagnosis of neonatal sepsis. Method: A study was carried out from January 2015 to December 2015 in hospital, based on, samples of babies with history of infective etiology within the first 28 days of birth. Result: One hundred neonatal cases of clinically suspected septicemia were studied. Culture positive rate was $26 \%$. E coli was the major organism responsible for early onset of sepsis. The highest sensitivity and negative predictive value was found for septic score of more than or equal to 3 by septic scoring, I:T ratio and C- reactive protein with the highest sensitivity, micro ESR and TLC with the highest specificity. Conclusion: CRP emerged as the test with the highest sensitivity. Micro- ESR and TLC had the highest specificity.
\end{abstract}

Keywords: Neonatal sepsis, Blood culture, C- reactive protein, Micro ESR.

\section{Introduction}

Neonatal sepsis in neonates is a clinical syndrome characterized by systemic sign of infection accompanied by bacteremia in the first 28 days of birth [1]. Neonatal sepsis is a complex syndrome caused by an uncontrolled systemic inflammatory response of infectious origin characterized by multiple manifestations which can result in dysfunction and failure of one or more organs and even death [2]. Neonatal sepsis is one of the most important, commonest and major causes of morbidity and mortality. The incidence was 3\% among the intramural births, with an overall mortality rate variation of $18.6 \%$ [3]. Among the extramural ad mission, sepsis was responsible for $39.7 \%$; on the other hand, indiscriminative overuse of antibiotics, was hazardous for any neonatal unit which may lead to overtreatment

Manuscript received: $28^{\text {th }}$ May 2016

Reviewed: $12^{\text {th }}$ June 2016

Author Corrected; $26^{\text {th }}$ June 2016

Accepted for Publication: $10^{\text {th }}$ July 2016 and emergence of resistant organism [4, 5]. The clinicians focused on diagnostic test which has high sensitivity and high negative predictive accuracy. These are direct or indirect hematological tests [5]. Septic screens are the batteries of parameter studies in order to improve diagnostic accuracy [5]. Of these, leukocyte count and indices of micro ESR and CRP were the most studied tests which were cost efficient. Therefore, a group of tests was studied to access the usefulness either singly or in combination with predicting neonatal sepsis. These tests were of total leucocytecount (TLC), Absolute neutrophil count (ANC) immature total ratio (I:T ratio) platelet count, micro ESR and CRP.

\section{Materials and Methods}

Astudy was conducted in the neonatology unit of Princess Esra Hospital attached to Deccan College of Medical Sciences, Hyderabad, Telangana State, India from $1^{\text {st }}$ January 2015 to end of December 2015. In all, 
100 neonates were studied. Samples included babies with history of illness of ineffective etiology with the onset within 28 days of birth.

Inclusive Criteria : Neonate intramural or extramural delivery, clinically presenting symptoms of sepsis on physical examination such as refusal to feed, lethargy, hypothermia, hyperthermia, vomiting, abdominal distension and diarrhea were studied.

Exclusive Criteria: Neonates with lethal congenital anomalies, extreme low birth weight, respiratory distress syndrome and babies treated previously with antibiotics for more than 12 hours were excluded from this study. Detailed clinical examination of each baby was carried out. Gestational age was also calculated. When illness started in the first 72 hours of birth,it was an early onset of disease and after 72 hours, it was late onset. Gestational age of babies was assessed using Ballard Scoring system to classify them as preterm, term, and post term. Blood samples were collected from the neonates suspected for septicemia. The study was divided into three groups for analysis as follows; Proven sepsis, probable sepsis, and no sepsis. Proven sepsis was defined as blood culture or CSF culture or culture from other significant site showing positive for any bacterial growth within 48 to 72 hours. Investigation suggested sepsis. $1 \mathrm{ml}$ of blood sample was collected through peripheral venipuncture in a bottle containing Potassium EDTA. It was subjected to platelet count. Total leucocyte count and differential count analysis were shown on automated cell counters. Band forms with less mature cellswere classified as immature polymorpho nuclear leucocytes. I:T ratio was calculated after counting 200 cells. Total leucocytes count, and absolute neutrophil count was recorded .

I : T ratios tested as Monroe's criteria was for term babies and mouzinho criteria for VLBW babies. $0.5 \mathrm{ml}$ to $1 \mathrm{ml}$ of blood sample was taken for micro ESR and CRP estimation. Venous blood was collected in preheparinized microhemocrit glass tube of $7.5 \mathrm{~mm}$ length. More than or equal to $15 \mathrm{~mm}$ at the end of first hour was taken as significant. C Reactive protein was done by semi quantitative method using Rhelax CRP kit. A positive agglutination with undiluted serum sample corresponding to CRP concentration of 6 micro $\mathrm{g} / \mathrm{ml}$ was used to calculate each parameter and combination of parameters. Statistical analysis was done using graph pad prism 5 (Graphpad software, Inc, USA). Data is presented as mean $+\mathrm{SD}$.

Variable difference with $\mathrm{P}<0.05$ is considered statistically significant. The sample size was determined by using the open EPI statistics of $95 \%$ confidence to detect the result with $90 \%$ of sample power.

\section{Results}

One hundred neonates admitted in the neonatal unit, were divided into Group A and Group B. In group A, the neonates were subjected to CBP which included TLC, ANC, I:T ratio, platelet count, micro ESR, CRP, blood culture, and other investigations to support diagnosis. In group B, all investigations were carried out other than blood culture to support diagnosis. The babies were subjected to sepsis in the first 28 days of birth. The study showed that the group comprised 61 males and 39 females in the ratio of 1.56:1

Table -1: Hematological Investigation in relation to blood culture.

\begin{tabular}{|c|c|c|c|c|c|}
\hline \multirow[t]{2}{*}{ Test } & \multicolumn{2}{|c|}{ Positive } & \multirow{2}{*}{$\begin{array}{l}\text { Negative } \\
\text { Culture } \\
\text { Positive }\end{array}$} & \multirow[b]{2}{*}{$\begin{array}{c}\text { Culture } \\
\text { Negative }\end{array}$} & \multirow[b]{2}{*}{$\mathbf{k}$} \\
\hline & $\begin{array}{l}\text { Culture } \\
\text { Positive }\end{array}$ & $\begin{array}{l}\text { Culture } \\
\text { Negative }\end{array}$ & & & \\
\hline TLC & 08 & 08 & 05 & 29 & 0.40 \\
\hline ANC & 10 & 15 & 15 & 10 & 0.28 \\
\hline I:T Ratio & 13 & 12 & 00 & 25 & 0.52 \\
\hline Platelets & 02 & 09 & 11 & 28 & 0.00 \\
\hline Micro ESR & 07 & 08 & 06 & 29 & 0.30 \\
\hline CRP & 11 & 18 & 02 & 19 & 0.26 \\
\hline 2 or more & 12 & 18 & 01 & 19 & 0.30 \\
\hline 3 or more & 12 & 09 & 01 & 28 & 0.57 \\
\hline
\end{tabular}

Table- 1: shows that total leucocyte count suggestive of sepsis i.e positive was found in 16 cases. .Of these, 8 cases were bacteriologically positive and of the 34 negative cases, 5 grew organism on culture. I:T ratio of more than 0.2 was found 
in 25 cases of which 13 were positive bacteriologically whereas, in 25 negative cases, none was culture positive. ANC was positive in 25 cases of which 10 were proven. Platelet count was less than 1,50,000 per cu.mm in 11 cases of which 2 cases were culture proven. Micro ESR was significantly increased in 15 cases and it was negative in 35 cases of which 6 grew organism I:Tratio was the most sensitive test. CRP was the second most sensitive test being positive in 29 cases, out of which 11 were bacteriologically proven cases. I:T ratio of more than 0.2 and CRP more than 0.6 microgram $/ \mathrm{ml}$ emerged as the most sensitive tests, where micro ESR was more than $15 \mathrm{~mm}$ in first hour. TLC and platelet count for less than $1,50,000 / \mathrm{cu} \mathrm{mm}$ was the most specific test.

Table- 2: Accuracy of Hematological Parameters.

\begin{tabular}{|c|c|c|c|c|c|c|c|}
\hline Test & $\begin{array}{c}\text { Total } \\
\text { Positive }\end{array}$ & $\begin{array}{c}\text { Positive with } \\
\text { Proven Sepsis }\end{array}$ & Sn & Sp & PPV & NPV & LR \\
\hline TLC & 16 & 08 & 62 & 78 & 50 & 85 & \\
\hline ANC & 25 & 10 & 77 & 59 & 40 & 88 & \\
\hline I:T Ratio & 25 & 13 & 100 & 68 & 52 & 100 & \\
\hline Platelets & 11 & 02 & 15 & 76 & 18 & 72 & 78 \\
\hline Micro ESR & 15 & 07 & 54 & 78 & 47 & 90 & \\
\hline CRP & 29 & 11 & 85 & 51 & 38 & 95 & \\
\hline $\begin{array}{c}2 \text { or more test } \\
\text { positive }\end{array}$ & 30 & 12 & 92 & 51 & 40 & 56.6 & \\
\hline $\begin{array}{c}3 \text { or more test } \\
\text { positive }\end{array}$ & 21 & 12 & 92 & 76 & 57 & 96.6 \\
\hline
\end{tabular}

Table- 2 Shows that the proven cases of Sepsis are taken as positive and other cases are taken as negative. Sensitivity of Septic screen is $92 \%$, Specificity is $51 \%$, PPV is $40 \%$ and most importantly $95 \%$ of the negative predictive accuracy, the prevalence of the proven sepsis is considered as $26 \%$.

Table- 3: Performance of various combination parameters.

\begin{tabular}{|c|c|c|c|c|c|c|}
\hline Combination of Parameters & Total Positive & $\begin{array}{l}\text { Positive with } \\
\text { Proven }\end{array}$ & Sn & Sp & PPV & NPV \\
\hline \multicolumn{7}{|l|}{ Two Test } \\
\hline ANC + I:T Ratio & 17 & 10 & 77 & 81 & 59 & 91 \\
\hline $\mathrm{TLC}+\mathrm{PC}$ & 3 & 2 & 15 & 97 & 67 & 77 \\
\hline I:T Ratio + CRP & 23 & 11 & 85 & 68 & 48 & 93 \\
\hline \multicolumn{7}{|l|}{ Three Test } \\
\hline TLC+PC+ I:T Ratio & 3 & 2 & 15 & 97 & 66 & 77 \\
\hline ANC + I:T Ration + CRP & 15 & 11 & 85 & 89 & 73 & 94 \\
\hline I:T Ratio + Micro ESR+ CRP & 13 & 8 & 62 & 86 & 62 & 86 \\
\hline \multicolumn{7}{|l|}{ Four Test } \\
\hline TLC+PC+CRP+I:T Ratio & 3 & 2 & 15 & 97 & 66 & 77 \\
\hline \multicolumn{7}{|l|}{ Five Test } \\
\hline $\begin{array}{c}\text { TLC+ANC+ I:T Ratio + } \\
\text { CRP+Micro ESR }\end{array}$ & 7 & 4 & 31 & 92 & 57 & 79 \\
\hline $\begin{array}{c}\text { TLC+ANC+I:T Ratio+CRP+ } \\
\text { Platelets }\end{array}$ & 2 & 1 & 08 & 97 & 50 & 75 \\
\hline \multicolumn{7}{|l|}{ All Six Test } \\
\hline $\begin{array}{c}\text { TLC+ANC+I:T Ratio } \\
+ \text { CRP+MicroESR+Platelet Count }\end{array}$ & 1 & 0 & 00 & 97 & 00 & 73 \\
\hline
\end{tabular}

Table 3: Shows that of the two test combination, I:T ratio+CRP emerged as most sensitive tests. It identified 11 out of 23 cases of sepsis of $85 \%$ out of the two and also a reasonably good specificity of $69 \%$. TLC + PC emerged as the most specific test combination giving specificity of $97 \%$. ANC + I:T ration + CRP were the best in three test combinations 
with $85 \%$ sensitivity and $89 \%$ specificity. TLC $+\mathrm{PC}+\mathrm{I}$ :T ratio produced a specificity of $97 \%$. TLC + I:T ratio $+\mathrm{CRP}+\mathrm{PC}$, these four test combinations had a good specificity of $97 \%$. Five and six test combinations identified almost all infants with sepsis.

\section{Discussion}

The present study was carried out to study rapid diagnosis of septicemia in newborn babiesupto first month (28days of life) by using some indirect hematological tests with reference to age and dependent neutrophil indices. The prevalence of neonatal sepsis was $26 \%$ which was comparable with the studies of Philip et al [6] and Gerdes et al [5, 6]. Early diagnosis of neonatal sepsis was found to have prevalence of $7.9 \%$ and $5.9 \%$ respectively which is much lower than what was observed in Singhet al [7] who observed itto be $27.8 \%$ in their study. Joshi et al [8] also reported prevalence of $25.8 \%$ which was also similar to these studies. In present study, sensitivity, specificity, positive predictive value, negative prediction value of laboratory parameters were evaluated in relation to blood culture taken as gold standard.

Table- 4: Total Leucocyte Count (TLC).

\begin{tabular}{|c|c|c|c|c|}
\hline Authors (Year) & SN\% & SP\% & PPV \% & NPV \% \\
\hline Philip et al [6] & 50 & 94 & 40 & - \\
\hline Rodwel et al [16] & 44 & 92 & 36 & 94 \\
\hline Basu et al [18] & 12 & 93 & 50 & 32 \\
\hline Present Study & 62 & 78 & 50 & 85 \\
\hline
\end{tabular}

In Present study TLC has a sensitivity of $61 \%$ comparable to Philip et al (6) $\{50 \%\}$ and more than that reported by Rodwel et al [16] (44\%). Specificity of 78\% observed in the present study was more than that reported by Namdeo et at [17] (69\%) and less than that of Mishra et al [13] which is (88\%). The positive predictive value of 50\% observed in the present study is equivalent to the value reported by Basu et al [18]. High negative predictive value of $85 \%$ was observed in the present study which was comparable with observations made by Rodwel et al [16] and Philip et al [6]. The difference of these studies, when individual predictors of sepsis are considered varied in positive test criteria. I:T ratio was found to have higher sensitivity of $10 \%$ which is equivalent to Gerdes et al [5] which is (100\%) and comparable to Rodwel et al [6] which is (96\%) and Basu et al [18] which is (92\%). Philip et al [6]. Mishra et al [13] studied band cell to neutrophil ratio and found to have lower sensitivity as compared with I:T ratio i. e $90 \%$ and $92 \%$ respectively. Specificity of $68 \%$ observed in the present study was comparable with $71 \%$ as observed by Rodwell et al [16] which is (44\%). Specificity of $78 \%$ observedin the present study was more than that reported by Namdeo et al [17] as which is less than that reported by Mishra etal [13] which is (88\%). Positive predictive value of 50\% observed in the present study is equivalent to Basu etal [18]. High negative predictive value of $85 \%$ observed in the present study was comparable with observation made by Rodwelet al [16] philip et al [6]. I: T ratio was found to have higher sensitivity of $100 \%$ which is equivalent to Gerdes et al [5] which is (100\%) and comparable to Rodwel et al [16] which is (96\%) and Basu et al which is [18] (92\%). Philip et al [6], Mishra et al [13] studied band cells to neutrophil ratio and found to have lower sensitivity as compared with I : T ratio i.e $90 \%$ and $92 \%$ respectively. Specificity of $68 \%$ observed in the present study was comparable with $71 \%$ as observed by Rodwel te al [16], which is higher than reported Gerdes et al [5]. Most importantly I:T ratio has got the highest negative predictive value amongst all neutrophils indices i.e of $100 \%$.

Table-5: Micro ESR

\begin{tabular}{|c|c|c|c|c|}
\hline Author (year) & Sn(\%) & SPC\% & PPYC\% & NOVC \\
\hline Philipe et al [6] & 30 & 97 & 43 & - \\
\hline Gerdes et al [5] & 31 & 94 & 24 & 95 \\
\hline Singh et al [7] & 55 & 81 & - & - \\
\hline Present study & 54 & 78 & 47 & 78 \\
\hline
\end{tabular}

(Table: - 5 Shows that Micro- ESR was found to have specificity more than sensitivity, of $78 \%$ over $54 \%$ reported in the present study which was comparable with that observed by Singh etal [7] which was 81\%, and Gerdes et al [5] which was $94 \%$ of specificity, Philip et al [6] was reported $97 \%$ of specificity and $30 \%$ of sensitivity. Singh et al [7] observed sensitivity of $55 \%$ which is comparable with the present study value of $54 \%$ of sensitivity. 
Table-6: $\mathrm{C}$ reactive Protein.

\begin{tabular}{|c|c|c|c|c|}
\hline Author (year) & Sn(\%) & SPC\% & PPYC\% & NOVC \\
\hline Phillipe et al [6] & 47 & 86 & 22 & - \\
\hline Singh et al [7] & 80 & 91 & - & - \\
\hline Gerdes et al [5] & 93 & 85 & 28 & 99 \\
\hline Basu et at [18] & 92 & 68 & 92 & 96 \\
\hline Present study & 85 & 51 & 38 & 90 \\
\hline
\end{tabular}

CRP was found to have the highest sensitivity in the tests other than neutrophils indices i.e. I:T ratio in the present study. The observation is similar to Singh et al [7] and comparable with Gerdes et al [5] and Basu et al [18]. It has the specificity of $51 \%$ which is less than that of Basu et al [18] High negative predictive value of $90 \%$ was found in the study of CRP which is comparable with Basu et al [18] and lower than reported by Gerdes et al [5] . CRP test was a good indicator with high sensitivity and affected by other factors like both weight, gestational age and onset of disease. In the present study two or more test positives had sensitivity of $92 \%$, Specificity of $51 \%$, positive and negative predictive value of $40 \%$ and $95 \%$ respectively which are comparable with that of Philip etal [6] who showed the sensitivity of $93 \%$. Specificity- $88 \%$ and positive predictive value as 39\%. These Values were also comparable with 3 of Singh et al [7] $86 \%$ of sensitivity and $90 \%$ specificity. Amongst two test combinations I: T ratio + CRP emerged as the most sensitive (85\%) and with specificity (68] and TLC + PC emerged as the most Specific test with specificity of $97 \%$ in two test combinations. Sharma et al [11], reported CRP + B:N ratio of having sensitivity $100 \%$ and specificity $51.7 \%$. Singh et al [7] reported ANC $+\mathrm{CRP}$ as the most specific one, whereas Philip et al [6], reported ANC +BN: TN as the most useful combination with highest positive predictive value. Sharma et al [11], reported CRP+ micro ESR + Toxic generation, as the most useful in three test combinations with sensitivity of $100 \%$ and specificity of $82 \%$ which is comparable with the present study. The present study had higher number of low birth weight babies as $80 \%$, out of which $22 \%$ were below1500 gm weight. These observations were similar to those reported by Sharma et al [11] (70\%), Ahmed et al [10] $(60 \%)$ and Joshi et al [8]. However, they reported $116(50.4 \%)$ as low birth weight cases in their study.The present study had $60 \%$ cases of early onset sepsis while cases with late onset septicemia were $40 \%$. Sharma et al [13] observed 33 cases $(66 \%)$ of more than 7 days of age. Incidences of refusal to feed was comparable with Guha et al [7] who observed it to be $66.25 \%$ higher than reported by Ahmed et al [10] (27\%) and Waber et al [12] as 41.5\%. Lethargy and respiratory distress cases were seen as $80 \%$ and $44 \%$ respectively in the present study. Guha et al [7] and Sharma et al [11] reported similar incidences of lethargy as $61.25 \%$ and $60 \%$ cases respectively. Hypothermia was found in 10 cases in the present study, which were preterm babies indicating that hypothermia was more common in preterm babies with septicemia. Chandana et al [14] reported 14\% incidences of hyperthermia and $12 \%$ incidences of hypothermia.

Abdominal distension and vomiting were seen in 13 and 6 cases respectively whereas diarrhea was found in $9 \%$ babies. These findings were similar to those reported by Mishra et al [13]. In the present study, the total leucocyte count sensitivity was $61 \%$ comparable with Philip et al $50 \%$ and more than that reported by Rodwell et al [16]. Specificity of $78 \%$ observed in the present study was more than that reported by Namdeo et al [17] as $69 \%$ and less than that of Mishra et al [13] which is $88 \%$ positive predictive value of $50 \%$ observed in the present study was equivalent to Basu et al [13]. In the present study, platelet count $\left(<1,50,000 / \mathrm{mm}^{3}\right)$ emerged as the test with good specificity but poor sensitivity. It was found to have specificity of $76 \%$ which was comparable with that reported by Mishra et al ([13] 79\% and Basu et al [18], as $71 \%$. Micro ESR was found to have specificity more than sensitivity, of $78 \%$ over $54 \%$ reported in the present study which was comparable with that observed by Singh et al [22] which $81 \%$ and Gerdes et al [21] which is $94 \%$. In the present study, 100 cases were enrolled on clinical suspicion of sepsis.

Cultural positivity rate was $26 \%$ which was similar to that of Singh et al [ 22] 28\% Joshi et al [8] which was $25.8 \%$. In the present study, staphylococcal sp. were the commonest isolates 4/13 along with E coli 4/13 cases followed by klebsiella in 3/13 cases. Blood culture was positive in 12 cases with a blood culture positivity rate of $24 \%$. Ecoli was the commonest organism implicated for early onset sepsis in the present study i.e. $43 \%$. staph sp 50\% was the commonest organism responsible for late onset of sepsis, klebsiella spp 33\% and Ecoli 17\% were also responsible with $43 \%$ cases of early onset of sepsis. In the present study, 23.3\% Boo et al [23] was reported as the highest mortality associated with Klebsiella Sp of $63.8 \%$ followed by Ecoli in $57.7 \%$ and staph $27.3 \%$, but in the present study, there was no mortality related to Klebsiella $\mathrm{Sp}$. Ahmed et al [10] studied 30 cases of which 12 cases expired overall mortality and case fatality 
rate was $28 \%$ in the present study. Karthikeyan [24] was observed as case fatality rate of $13.5 \%$ which was lower than the present study Joshi et al [8] observed mortality of 32.2\% which was higher than the present study. Mortality amongst culture positive cases in present study was 31\% which was lower as compared with that of Mishra et al [25] who observed mortality of $61.7 \%$. Among gram negative septicemia $71 \%$ died whereas mortality was only $49 \%$ in gram positive septicemia.

\section{Conclusion}

Neonatal septicemia is a life threatening emergency Blood culture positive rate was $26 \%$. E.coli was the major organism responsible for early onset of sepsis and staph sp. Was the common culprit for late onset of sepsis. Highest sensitivity and negative predictive values were found for a septic score of more than or equal to 3 by septic scoring. For prevention of neonatal septicemia, it requires to improve survival rate, better approach with early initiation of appropriate antibiotics.

It also requires infection control policies at the national level for effective management of such infections. To define the need for antibiotic therapy, several tests recommended as helpful in diagnosis neonatal sepsis were evaluated.

\section{Funding: Nil, Conflict of interest: Nil Permission from IRB: Yes}

\section{References}

1. Jerome ok, Marcy SM. Bacterial sepsis and meningitis in infectious diseases of fetus and newborn infants $4^{\text {th }}$ edl. Jack. S. Remington, Jerome o.klein edition W.B sounders. Philadelpia 1995pp 836-40.

2. AnupamSachder, Sunil Sarin, Neonatal Sepsis, Jounal of P.G. Medical Education and Training and Research 36, Vol. 11 No. 1 Jan-Feb 07.

\section{National Neonatal and PerinalDatabase, 2002-03.}

4.Paul VK, Singh M. Diagnosis and treatment of neonatal sepsis. Indian Pediatr. 1986 Dec;23(12): 1023-35.

5. Gerdes JS, Polin R. Early diagnosis and treatment of neonatal sepsis. Indian J Pediatr. 1998 Jan-Feb; 65(1):63-78.

6. Philip AG, Hewitt JR. Early diagnosis of neonatal sepsis. Pediatrics. 1980 May;65(5):1036-41.

7. Singh M, Narang A, Bhakoo ON. Evaluation of a sepsis screen in the diagnosis of neonatal sepsis. Indian Pediatr. 1987 Jan;24(1):39-43.
8. Joshi SG, Ghole VS, Niphadkar KB. Neonatal gramnegative bacteremia. Indian J Pediatr. 2000 Jan; 67 (1): 27-32.

9. Tallur SS, Kasturi AV, Nadgir SD, Krishna BV. Clinico-bacteriological study of neonatal septicemia in Hubli. Indian J Pediatr. 2000 Mar;67(3):169-74.

10. Nawshad Uddin Ahmed ASM, Chaudhery A, Hogue M, Darmstadt Gill clinical and bacteriological profile of neonatal septicemia in a tertiary level pediatric hospital in Bangladesh Indian pediatric 2001:39:1034-9.

11. Sharma A, Kutty CV, Sabharwal U, Rathee S, Mohan H. Evaluation of sepsis screen for diagnosis of neonatal septicemia. Indian J Pediatr. 1993 Jul-Aug; 60(4):559-63.

12.Rodrigvez- Weber MA, Lopez-Candianic Arrendonlo - Garcia JL el al, Morbidity and morbidity from neonatal bara sepsis in a tertiary care level hospital saludpublica de Nex.co 2003;45(2):1.8.

13. Misra PK, Kumar R, Malik GK, Mehra P, Awasthi S. Simple hematological tests for diagnosis of neonatal sepsis. Indian Pediatr. 1989 Feb;26(2):156-60.

14. Chandana A, Rao NM, Shriniwas M Shyamala S. Rapid diaggonitive tests in neonatal septicemia. Indian. J pedletr 1988,55(5) : 947-50.

15. Bhakoo ON, Singh M. Perinatal risk factors in neonatal bacterial sepsis. Indian J Pediatr. 1988 NovDec;55(6):941-6.

16. Rodwell RL, Leslie AL, Tudehope DI. Early diagnosis of neonatal sepsis using a hematologic scoring system. J Pediatr. 1988 May;112(5):761-7.

17. Namdeo UK, Singh HP, Rajput VJ, Kushwaha JS. Hematological indices for early diagnosis of neonatal septicemia. Indian Pediatr. 1985 Apr;22(4):287-92.

18. Basu S. Gelruprasad,Narang 4, Garcusal G. 
Diagnosis of Sepsis in the high risk neonate using a hematology scoring System . Indian J Hemat Blood Transf 1999, 17(2) 32-4.

19. Kite $\mathrm{P}$, Millar MR, Gorham $\mathrm{P}$, Congdon $\mathrm{P}$. Comparison of five tests used in diagnosis of neonatal bacteraemia. Arch Dis Child. 1988 Jun;63(6):639-43.

20. Kuruvilla KA, Pillai S, Jesudason M, Jana AK. Bacterial profile of sepsis in a neonatal unit in south India. Indian Pediatr. 1998 Sep;35(9):851-8.

21. Gerdes JS, Polin RA. Sepsis screen in neonates with evaluation of plasma fibronectin. Pediatr Infect Dis J. 1987 May;6(5):443-6.
22. Singh M, Narang A, Bhakoo ON. Evaluation of a sepsis screen in the diagnosis of neonatal sepsis. Indian Pediatr. 1987 Jan;24(1):39-43.

23. Boo NY, Chor CY. Six year trend of neonatal septicaemia in a large Malaysian maternity hospital. J Paediatr Child Health. 1994 Feb;30(1):23-7.

24. Karthikeyan G, Premkumar K. Neonatal sepsis: Staphylococcus aureus as the predominant pathogen. Indian J Pediatr. 2001 Aug;68(8):715-7.

25. Mishra JN, Rai MG, Chakraborty S, Prasad S. Study of neonatal septicemia. Indian Pediatr. 1985 Apr; 22 (4):281-5.

\section{How to cite this article?}

Mir Sumsam Ali Khurram, Naseem A, Prasad C.N. D. A comparative study of CBP, CRP, Micro ESR and blood culture in the diagnosis of neonatal septicemia. Int $J$ Pediatr Res.2016;3(7):529-535.doi:10.17511/ijpr.2016.i07.12. 